\title{
A Tentorial Venous Hemangioma Presenting As an Extra-Axial Mass in the Ambient Cistern: A Case Report
}

\author{
Soichi Oya ${ }^{1,2}$ Richard A. Prayson ${ }^{3}$ Joung H. Lee ${ }^{1,2}$ \\ ${ }^{1}$ Brain Tumor and Neuro-Oncology Center, Neurological Institute, \\ Cleveland Clinic, Cleveland, Ohio, United States \\ Address for correspondence and reprint requests Joung H. Lee, M.D., \\ Brain Tumor and Neuro-Oncology Center, Cleveland Clinic, 9500 Euclid \\ Avenue, Desk S73, Cleveland, OH, United States 44195 \\ Clinic, Cleveland, Ohio, United States \\ (e-mail: leej@ccf.org).
}

${ }^{3}$ Department of Anatomic Pathology, Cleveland Clinic, Cleveland,

J Neurol Surg Rep 2012;73:37-40.

\begin{abstract}
Keywords

- ambient cistern

- extra-axial

- tentorium

- vascular tumor

- venous hemangioma

Although venous hemangiomas are one of the most common soft-tissue tumors, venous hemangiomas in the central nervous system are extremely rare. We present an unusual case of venous hemangioma originating from the interdural space of the tentorium. A 32-year-old woman was incidentally found to have extra-axial mass occupying the left ambient cistern. This tumor was observed for the first 4 years as it was completely asymptomatic. Surgical resection was later recommended when the tumor grew. The mass originated from between the two layers of the anteromedial tentorial incisura. There were no findings indicative of previous hemorrhage inside the mass. The matrix of the mass was firm and vascular, resembling a fibrous meningioma. Gross total resection was achieved without any neurological deficit. Pathological examination revealed a dense fibrous connective tissue with a proliferation of vessels marked by thickened walls. A spindle cell proliferation in the vessel walls did not stain with the antibody to S-100 protein. Movat stain demonstrated the venous character of the vessels. These results were histologically compatible with a venous hemangioma. Albeit extremely rare, a venous hemangioma, a distinct clinical and pathological entity from a venous angioma, can present an intracranial mass lesion.
\end{abstract}

\section{Introduction}

Venous hemangiomas are one of the most common softtissue tumors, ${ }^{1}$ representing $7 \%$ of all benign soft-tissue tumors. ${ }^{2}$ Histologically, they are characterized as the proliferation of vascular channels whose walls contain layers of smooth muscle. ${ }^{3-5}$ These tumors frequently occur in the skin and subcutaneous tissue, but rarely arise in the central nervous system (CNS). ${ }^{6}$ We describe an extremely rare case of a venous hemangioma presenting as an extra-axial mass in the ambient cistern.

received

August 6, 2011

accepted after revision

December 19, 2011

published online

May 17, 2012

\section{Case Report}

A 32-year-old woman with no past medical history experienced lightheadedness that persisted for a week. Her neurological examination was unremarkable and her symptom resolved in a week. However, a magnetic resonance imaging (MRI) revealed an extra-axial mass in the left ambient cistern. This appeared slightly hypointense on T1-weighted images, hyperintense on T2-weighted images, and heterogeneously enhanced on postcontrast images ( $\mathbf{- F i g}$. 1). Although the mass was attached to the tentorium, the lack of the apparent
Copyright $\odot 2012$ by Thieme Medical Publishers, Inc., 333 Seventh Avenue, New York, NY 10001, USA. Tel: +1(212) 584-4662.
DOI http://dx.doi.org/ 10.1055/s-0032-1312680. ISSN 2193-6358. 

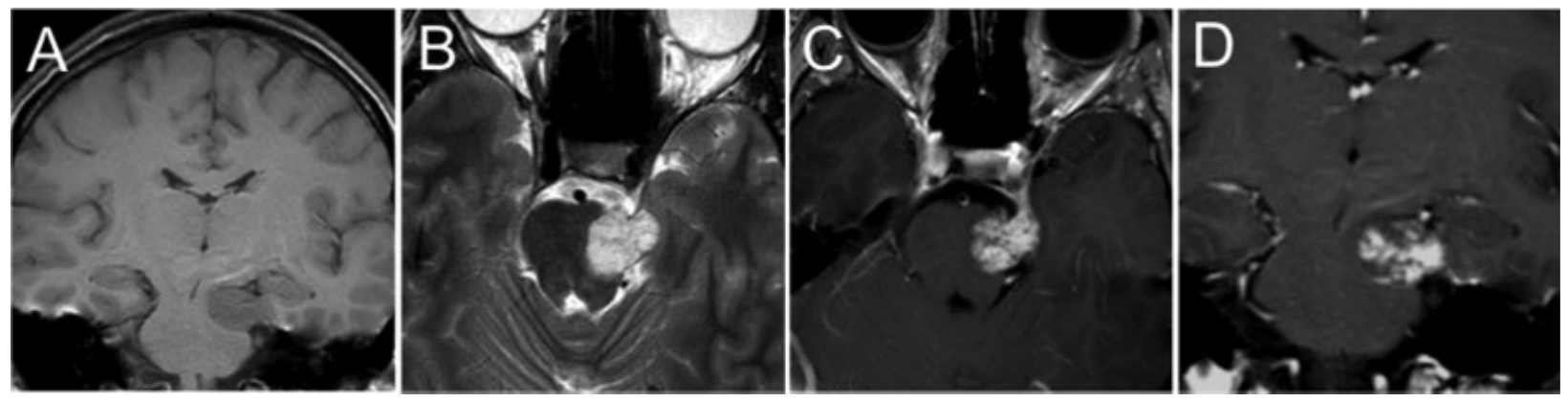

Figure 1 Preoperative magnetic resonance imaging scans. The coronal T1-weighted (A), axial T2-weighted (B), postcontrast axial, and coronal T1weighted (C, D) images showed an extra-axial mass with heterogeneous enhancement.

dural tail sign and the heterogeneous enhancement were thought to be atypical for meningiomas. The patient denied any symptoms including diplopia or abnormal facial sensation. Given its small initial size, the deep location and its incidental nature, a decision was made to observe the mass with serial imaging. The tumor remained stable for the first 3 years, but 4 years after the patient's initial presentation and MRI, it increased in size from the maximum diameter of 1.6 to $2.2 \mathrm{~cm}$. Possible therapeutic effects of gamma knife radiosurgery were discussed to control the growth, but surgical resection was subsequently recommended because of the atypical imaging features of the mass.

She underwent left temporal craniotomy followed by a subtemporal approach. The mass was identified as a very prominent bulge entirely contained within the tentorium, without any obvious exophytic component. This mass, covered by the medial aspect of the tentorium, was found to extend into the ambient cistern, causing significant compression on the adjacent cerebral peduncle. The center of the mass was entered by opening the superomedial tentorium. The tumor was noted to be arising from between the two layers of the anteromedial tentorial incisura. The fourth cranial nerve was displaced inferiorly by the mass. The anterior margin of the mass was just posterior to where the fourth nerve entered the cavernous sinus. The inferomedial surface of the cisternal portion of the tumor was covered by a thin capsule which was the inferior layer of the medial tentorium. No apparent adhesion to cranial nerves IV and V or the brainstem was observed. The mass had a fibrous and mildly vascular matrix, and had a gross appearance similar to a meningioma. There were no findings indicative of previous hemorrhage inside the mass. After aggressive central debulking, the mass was completely dissected off of the fourth and fifth cranial nerves, adjacent posterior cerebral and superior cerebellar arteries, and the brainstem. No apparent invasion to the posterior cavernous sinus was noted.

Her postoperative course was uneventful without any neurological deficit. Her MRI showed gross total resection (-Fig. 2). Pathological examination revealed dense fibrous connective tissue with an accompanying proliferation of vessels marked by thickened walls ( $\mathbf{- F i g .} \mathbf{3 A}$ ). A spindle cell proliferation in the vessel walls did not stain with antibody to S-100 protein. Intraoperative frozen section diagnosis was schwannoma. However, a Movat stain was subsequently performed which highlighted the venous nature of the back-to-back vessels (- Fig. 3B). These characteristics were compatible with a venous hemangioma. The patient was discharged 3 days after surgery without neurological deficits.

\section{Discussion}

The term "hemangioma" generally refers to benign vascular tumors that include a variety of clinically and histologically distinctive entities ${ }^{7}$ such as soft-tissue hemangiomas. The terminology is somewhat confusing because there have been many variations and overlaps in usage in the literature in reference not only to soft-tissue hemangiomas but also to cerebral vascular malformations. Soft-tissue hemangiomas are further classified as capillary, cavernous, arteriovenous, venous, and mixed according to which abnormal vascular channels are predominant. ${ }^{8,9}$ For example, a cavernous hemangioma is one of the pathological subtypes of soft-tissue hemangioma, but the term "cavernous hemangioma" overlaps with one type of cerebral vascular malformations. ${ }^{10}$ In

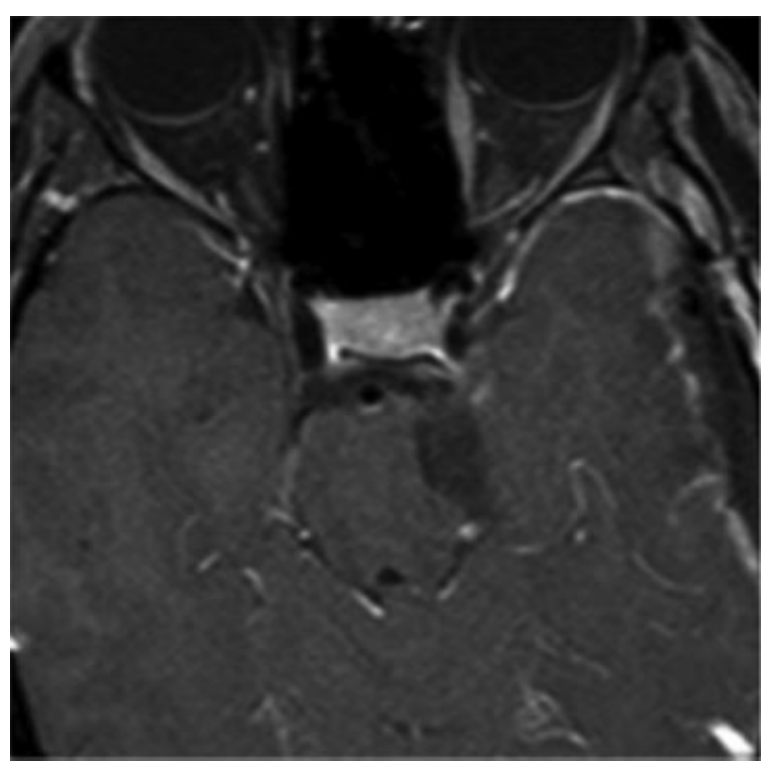

Figure 2 A postoperative magnetic resonance imaging scan showing gross total resection. 


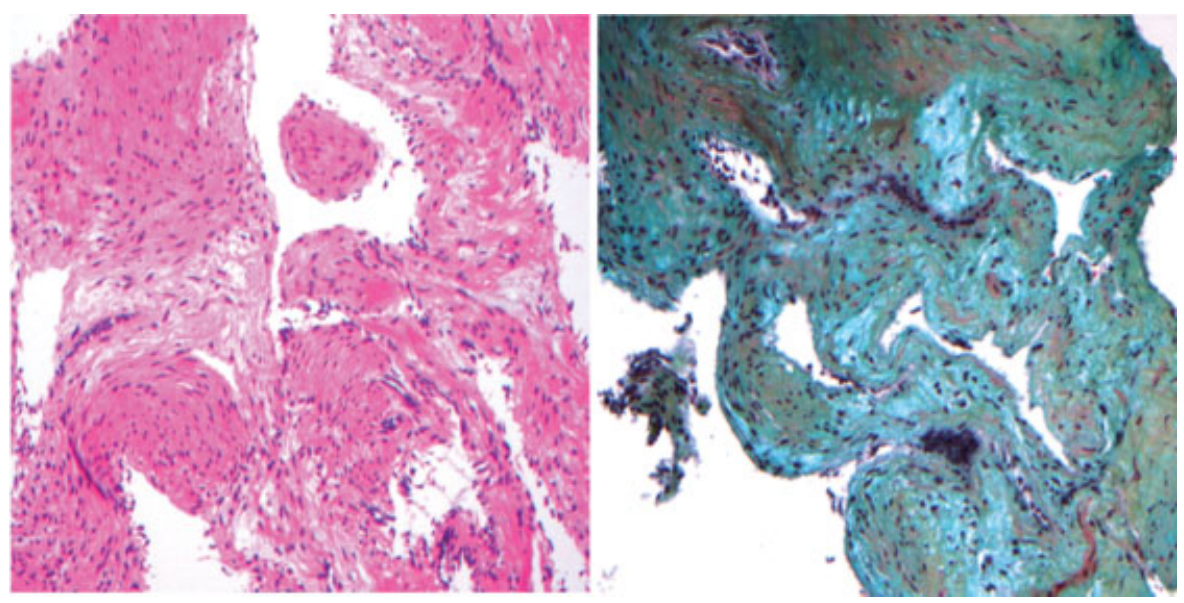

Figure 3 Left: A photomicrograph demonstrating dense fibrous connective tissue with a spindle cell proliferation of venous vessels marked by thickened walls. Hematoxylin and eosin stain, original magnification $\times 100$. Right: A Movat stain showed the venous nature of the back-to-back vessels in the specimen. Original magnification $\times 100$.

addition, although a venous angioma is distinct from a "venous hemangioma," some previous reports used the terms of a "venous angioma" and a hamartomatous "venous hemangioma" and interchangeably. ${ }^{4}$ Venous angiomas are currently called developmental venous anomalies. ${ }^{11}$ The classic description of the venous angioma is a cluster of venous radicles that converge into a collecting vein. ${ }^{12}$ They are intraparenchymal lesions with intervening brain tissue, depicted as a sunburst pattern on MRI or angiography. In our report, we use the term "venous hemangioma" as a hamartomatous proliferation of venous vessels with the thickened wall presenting a mass lesion.

Venous hemangiomas can arise in various parts of the body. They are frequently seen in skin, subcutaneous tissue, and skeletal muscle. Also some rare locations such as the mediastinum $^{13}$ or retroperitoneal cavity ${ }^{14}$ have been reported. Venous hemangiomas in the CNS are exceedingly rare. Our literature search found only four cases of "venous hemangiomas" in the CNS. Fermaglich et al $^{15}$ reported a case of surgical resection of a large enhanced globular mass in the optic chiasm that caused a rapid visual deterioration and subarachnoid hemorrhage. The mass contained patches of bluish discoloration and numerous blue cysts, implying previous hemorrhage. They concluded this lesion to be a venous angioma based on the microscopic findings of the numerous irregular vascular channels without the internal elastic membrane. Moore et $\mathrm{al}^{6}$ described a case of a 1-cm-sized venous hemangioma in the internal auditory canal that caused a persistent hearing loss since 5 years before presentation. Pathological examination revealed thick-walled blood vessels with smooth muscle and absence of elastic lamina. In the series of 426 surgical cases for tumors in the cerebellopontine angle and the internal acoustic canal, Kohan et $\mathrm{al}^{16}$ experienced two cases of vascular tumors, one of which was a venous hemangioma. The patient complained of rapidly progressive cochleovestibular symptoms. Monin et $\mathrm{al}^{4}$ described a case of a venous hemangioma of the left optic nerve sheath. The patient had left visual loss that was progressively worsening over a course of 5 months. The tumor was located underneath the optic nerve sheath with the appearance of a purplish and fibrinous mass. Partial resection was performed and the patient's vision improved.

Signs of hemorrhage were not observed on either radiological or intraoperative findings in our case. Because of the scarcity of cases, the bleeding rate of venous hemangiomas is unknown. However, intraoperative findings of previous reports appear to indicate the possibility of frequent intralesional hemorrhage. In addition, it should be noted that the sudden onset or relatively rapid progression of symptoms were observed in three of four previously reported cases. These clinical courses are uncharacteristic for small-sized benign tumors, which might also suggest that the intralesional bleeding could be seen in venous hemangiomas. Besides, there has been no report regarding the natural history of this rare lesion although the interval growth was noted during the follow-up (of 4 years) in our case.

From a surgical point of view, a good margin for dissection was obtained in our case, as was also described in previous reports. Similar to more common tumors in this location, such as schwannomas and meningiomas, the total resection seems to be feasible without injury to the brainstem and cranial nerves.

The information regarding the incidence, natural history, pathogenesis, and treatment options for venous hemangiomas in the CNS is currently unknown. Although the need for further accumulation of similar cases is obvious to provide the above information, for extra-axial lesions that show some atypical radiological characteristics and clinical courses, a venous hemangioma should be included in the differential diagnoses.

Finally, our differential diagnosis during the patient's observation included a schwannoma, and therefore, gamma knife radiosurgery was briefly discussed as a possible management option. This case underscores the importance of obtaining a tissue diagnosis before gamma knife radiosurgery. 
40 Venous Hemangioma in the Tentorium Oya et al.

\section{References}

1 Simon MA. Surgery for Bone and Soft Tissue Tumors. Philadelphia: Lippincott-Raven; 1998;534

2 Murphey MD, Fairbairn KJ, Parman LM, Baxter KG, Parsa MB, Smith WS. From the archives of the AFIP. Musculoskeletal angiomatous lesions: radiologic-pathologic correlation. Radiographics 1995; 15(4):893-917

3 Allen PW, Enzinger FM. Hemangioma of skeletal muscle. An analysis of 89 cases. Cancer 1972;29(1):8-22

4 Monin DL, Blumner K, Cohen NA, Brooks JS, Chen C, Kennedy DW. Endoscopic resection of a venous hemangioma of the optic nerve sheath. Ear Nose Throat J 2005;84(9):586, 588, 590 passim

5 Nichols GE, Gaffey MJ, Mills SE, Weiss LM. Lobular capillary hemangioma. An immunohistochemical study including steroid hormone receptor status. Am J Clin Pathol 1992;97(6): 770-775

6 Moore GF, Johnson PJ, McComb RD, Leibrock LG. Venous hemangioma of the internal auditory canal. Otolaryngol Head Neck Surg 1995;113(3):305-309

7 Benoit MM, North PE, McKenna MJ, Mihm MC, Johnson MM, Cunningham MJ. Facial nerve hemangiomas: vascular tumors or malformations? Otolaryngol Head Neck Surg 2010;142(1): $108-114$
8 Greenspan A, McGahan JP, Vogelsang P, Szabo RM. Imaging strategies in the evaluation of soft-tissue hemangiomas of the extremities: correlation of the findings of plain radiography, angiography, CT, MRI, and ultrasonography in 12 histologically proven cases. Skeletal Radiol 1992;21(1):11-18

9 Draud LA, Fix CF, eds. Vascular and Lymphatic Tumors. Philadelphia: Saunders; 2002;4186

10 McCormick WF, Schochet SS. Atlas of Cerebrovascular Disease. Philadelphia: Saunders; 1976

11 Lasjaunias P, Burrows P, Planet C. Developmental venous anomalies (DVA): the so-called venous angioma. Neurosurg Rev 1986;9 (3):233-242

12 Ruíz DS, Yilmaz H, Gailloud P. Cerebral developmental venous anomalies: current concepts. Ann Neurol 2009;66(3):271-283

13 Abe K, Akata S, Ohkubo Y, et al. Venous hemangioma of the mediastinum. Eur Radiol 2001;11(1):73-75

14 Igarashi J, Hanazaki K. Retroperitoneal venous hemangioma. Am J Gastroenterol 1998;93(11):2292-2293

15 Fermaglich J, Kattah J, Manz H. Venous angioma of the optic chiasm. Ann Neurol 1978;4(5):470-471

16 Kohan D, Downey LL, Lim J, Cohen NL, Elowitz E. Uncommon lesions presenting as tumors of the internal auditory canal and cerebellopontine angle. Am J Otol 1997;18(3):386-392 